\title{
ESTIMATES OF LOWER ORDER DERIVATIVES OF VISCOUS FLUID FLOW PAST A ROTATING OBSTACLE
}

\author{
REINHARD FARWIG \\ Fachbereich Mathematik \\ Darmstadt University of Technology \\ D-64283 Darmstadt, Germany \\ E-mail: farwig@mathematik.tu-darmstadt.de
}

\begin{abstract}
Consider the problem of time-periodic strong solutions of the Stokes system modelling viscous incompressible fluid flow past a rotating obstacle in the whole space $\mathbb{R}^{3}$. Introducing a rotating coordinate system attached to the body yields a system of partial differential equations of second order involving an angular derivative not subordinate to the Laplacian. In a recent paper [2] the author proved $L^{q}$-estimates of second order derivatives uniformly in the angular and translational velocities, $\omega$ and $k$, of the obstacle, whereas the transport terms fails to have $L^{q}$-estimates independent of $\omega$. In this paper we clarify this unexpected behavior and prove weighted $L^{q}$-estimates of first order terms independent of $\omega$.
\end{abstract}

1. Introduction. Consider the Navier-Stokes equations modelling viscous flow past a rotating body $K \subset \subset \mathbb{R}^{3}$ with axis of rotation $\omega=\tilde{\omega} e_{3}=\tilde{\omega}(0,0,1)^{T}, \tilde{\omega}=|\omega|>0$, and with velocity $u_{\infty}=k e_{3}, k>0$, at infinity. Then the velocity $v$ and the presure $p$ satisfy the system

$$
\begin{aligned}
v_{t}-\nu \Delta v+v \cdot \nabla v+\nabla q & =\tilde{f} & & \text { in } \Omega(t), t>0, \\
\operatorname{div} v & =0 & & \text { in } \Omega(t), t>0, \\
v(y, t) & =\omega \wedge y & & \text { on } \partial \Omega(t), t>0, \\
v(y, t) \rightarrow u_{\infty} & \neq 0 & & \text { as }|y| \rightarrow \infty,
\end{aligned}
$$

with an initial value $v(y, 0)=v_{0}(y)$, with constant viscosity $\nu>0$ and external force $\tilde{f}$ in the time-dependent exterior domain $\Omega(t)=O_{\omega}(t) \Omega$; here $O_{\omega}(t)$ denotes the orthogonal matrix

$$
O_{\omega}(t)=\left(\begin{array}{ccc}
\cos |\omega| t & -\sin |\omega| t & 0 \\
\sin |\omega| t & \cos |\omega| t & 0 \\
0 & 0 & 1
\end{array}\right)
$$

2000 Mathematics Subject Classification: 35C15, 35Q35, 76D05, 76D99, 76 U05.

Key words and phrases: Oseen flow, rotating obstacles, weighted estimates.

The paper is in final form and no version of it will be published elsewhere. 
Introducing the new independent and dependent variables

$$
x=O_{\omega}^{T}(t) y, \quad u(x, t)=O_{\omega}^{T}(t)\left(v(y, t)-u_{\infty}\right), \quad p(x, t)=q(y, t),
$$

and linearizing, $(u, p)$ will satisfy the modified Stokes system

$$
\begin{aligned}
u_{t}-\nu \Delta u+k \partial_{3} u-(\omega \wedge x) \cdot \nabla u+\omega \wedge u+\nabla p & =f, \\
\operatorname{div} u & =0,
\end{aligned}
$$

in a time-independent exterior domain $\Omega \subset \mathbb{R}^{3}$ together with the initial-boundary condition $u(x, t)=\omega \wedge x-u_{\infty}$ for $x \in \partial \Omega, u(x, 0)=u_{0}, u \rightarrow 0$ as $|x| \rightarrow \infty$. In the stationary whole space case to be analyzed in this paper we are led to the elliptic equation

$$
-\nu \Delta u+k \partial_{3} u-(\omega \wedge x) \cdot \nabla u+\omega \wedge u+\nabla p=f, \quad \operatorname{div} u=0 \text { in } \mathbb{R}^{3}
$$

in which the term $(\omega \wedge x) \cdot \nabla u$ is not subordinate to $-\nu \Delta u$. Note that a stationary solution $(u, p)$ of $(2)$ is related to a time-periodic solution of (1).

In [2] the author proved a priori estimates of strong solutions $(u, p)$ of $(2)$ which are found in the homogeneous Sobolev spaces $\hat{W}^{2, q}\left(\mathbb{R}^{3}\right)^{3} \times \hat{W}^{1, q}\left(\mathbb{R}^{3}\right)$ where

$$
\hat{W}^{k, q}(\Omega)=\left\{u \in L_{\text {loc }}^{1}(\bar{\Omega}) / \Pi_{k-1}: \partial^{\alpha} u \in L^{q}(\Omega) \text { for all } \alpha \in \mathbb{N}_{0}^{n},|\alpha|=k\right\} .
$$

Here $\partial^{\alpha}=\partial_{1}^{\alpha_{1}} \cdot \ldots \partial_{n}^{\alpha_{n}}$ for a multi-index $\alpha=\left(\alpha_{1}, \ldots, \alpha_{n}\right) \in \mathbb{N}_{0}^{n}$ and $\Pi_{k-1}$ denotes the set of all polynomials on $\mathbb{R}^{n}$ of degree $\leq k-1$. The space $\hat{W}^{k, q}(\Omega)$ consists of equivalence classes of $L_{\text {loc }}^{1}$-functions being unique only up to elements from $\Pi_{k-1}$ and is equipped with the norm $\sum_{|\alpha|=k}\left\|\partial^{\alpha} u\right\|_{q}$, where $\|\cdot\|_{q}$ denotes the $L^{q}$-norm. However, sometimes being less careful, we will consider $v \in \hat{W}^{k, q}(\Omega)$ as a function (representative) rather than an equivalence class of functions, i.e., $v \in L_{\text {loc }}^{1}(\Omega)$ such that $\partial^{\alpha} v \in L^{q}(\Omega)$ for every multi-index $\alpha$ with $|\alpha|=k$. For further results on similar problems we refer to [4], [8], [9], [10], [11], [12] and [13].

Theorem 1. (1) Let $1<q<\infty, \nu>0, k>0, \omega=(0,0, \tilde{\omega})^{T} \in \mathbb{R}^{3} \backslash\{0\}$, and let $f \in L^{q}\left(\mathbb{R}^{3}\right)^{3}$. Then the linear problem (2) has a solution $(u, p) \in \hat{W}^{2, q}\left(\mathbb{R}^{3}\right)^{3} \times \hat{W}^{1, q}\left(\mathbb{R}^{3}\right)$ satisfying the a priori estimate

$$
\left\|\nu \nabla^{2} u\right\|_{q}+\|\nabla p\|_{q} \leq c\|f\|_{q}
$$

with a constant $c$ independent of $\nu, k$ and $\omega$.

(2) Moreover,

$$
\left\|k \partial_{3} u\right\|_{q}+\|(\omega \wedge x) \cdot \nabla u-\omega \wedge u\|_{q} \leq c\left(1+\frac{k^{4}}{\nu^{2}|\omega|^{2}}\right)\|f\|_{q}
$$

with a constant $c>0$ independent of $\nu, k$ and $\omega$.

(3) Let $1<q<4, f \in L^{q}\left(\mathbb{R}^{3}\right)^{3}$ and let $(u, p) \in \hat{W}^{2, q}\left(\mathbb{R}^{3}\right)^{3} \times \hat{W}^{1, q}\left(\mathbb{R}^{3}\right)$ be the solution of (2). Then there exists $\beta \in \mathbb{R}$ such that

$$
\nabla^{\prime}(u-\beta \omega \wedge x) \in L^{r}\left(\mathbb{R}^{3}\right)^{6} \quad \text { for all } \quad r>1, \frac{1}{r} \in \frac{1}{q}-\left[\frac{1}{4}, \frac{1}{3}\right],
$$

and there exists a constant $C=C(\nu, k, \omega ; r)>0$ such that

$$
\left\|\nabla^{\prime}(u-\beta \omega \wedge x)\right\|_{r} \leq C\left(\|f\|_{q}+\left\|\nu \nabla g+(\omega \wedge x) g-k g e_{3}\right\|_{q}\right) .
$$


The proof of Theorem 1(1), see [2], is based on an explicit representation of $u$ the estimate of which uses Fourier transforms, Hardy-Littlewood decomposition methods and maximal operators. Estimate (4) shows a surprising and crucial dependence on $\frac{1}{|\omega|}$ via the term $\frac{k^{4}}{\nu^{2}|\omega|^{2}}$. On the one hand, it is not at all clear that the terms $k \partial_{3} u$ and $(\omega \wedge x) \cdot \nabla u-\omega \wedge u$ can be estimated in $L^{q}\left(\mathbb{R}^{3}\right)$ separately from each other. On the other hand, the dependence on $\frac{1}{|\omega|}$ seems to be unnatural. Note that, as $|\omega| \rightarrow 0$, problem (2) converges formally to Oseen's equation

$$
-\nu \Delta u+k \partial_{3} u+\nabla p=f, \quad \operatorname{div} u=0
$$

the solutions of which satisfy the estimate $\left\|k \partial_{3} u\right\|_{q} \leq c\|f\|_{q}$, see $[1,5]$. To be more precise, a sequence of solutions $\left(u_{\omega}\right)$ converges weakly in $\hat{W}^{2, q}\left(\mathbb{R}^{3}\right)^{3}$ to the solution of Oseen's equation as $|\omega| \rightarrow 0$, cf. [2] Remark 1.3(1).

Concerning Theorem 1(3) note that the solutions of the homogeneous system (2) are given by $\beta \omega \wedge x+\alpha e_{3}, \alpha, \beta \in \mathbb{R}$. Hence, with $u$ also $u-\beta \omega \wedge x$ is a solution of (2). The proof of Theorem 1(3) uses an improved Sobolev embedding theorem exploiting the fact that besides $\nabla^{2} u$ also $k \partial_{3} u$ lies in $L^{q}$, cf. [1]. However, the dependence on $1 /|\omega|$ in (4) implies that the constant $C$ in (5) also depends on $1 /|\omega|$. Due to this dependence the above-mentioned weak convergence of $\left(u_{\omega}\right)$ in $\hat{W}^{2, q}\left(\mathbb{R}^{3}\right)^{3}$, i.e. of second order derivatives in $L^{q}$, seems not to extend to $k \partial_{3} u_{\omega}$ in $L^{q}$.

The aim of this paper is to clarify these unusual features. In Theorem 2 below we present an improvement of (4) and simplify the proof given in [2]. Then, for small $q$, we prove a weighted $L^{q}$-estimate of $k \partial_{3} u$ and of $(\omega \wedge x) \cdot \nabla u-\omega \wedge u$ independent of $|\omega|$, see Theorem 4. An example and a heuristic argument will show that the dependence in (4) on $\frac{k}{\sqrt{\nu|\omega|}}$ is not a weakness of the proof.

Theorem 2. Let $1<q<\infty, \nu>0, k \in \mathbb{R}, \omega=(0,0, \tilde{\omega})^{T} \in \mathbb{R}^{3} \backslash\{0\}$ and let $f \in L^{q}\left(\mathbb{R}^{3}\right)^{3}$. Then the solution $u \in \hat{W}^{2, q}\left(\mathbb{R}^{3}\right)^{3}$ satisfies the a priori estimate

$$
\left\|k \partial_{3} u\right\|_{q}+\|(\omega \wedge x) \cdot \nabla u-\omega \wedge u\|_{q} \leq c\left(1+\frac{k^{2}}{\nu|\omega|}\right)^{2 \max (1 / q, 1-1 / q)+\varepsilon}\|f\|_{q}
$$

with a constant $c>0$ independent of $\nu, k$ and $\omega$; here $\varepsilon>0$ can be chosen arbitrarily small and $\varepsilon=0$ if $q=2$.

EXAmple 3. In Section 2 we will show that the term $\frac{k^{2}}{\nu|\omega|}$ is needed in the $L^{2}$-case. However, it is not clear whether the exponent $2 \max (1 / q, 1-1 / q)+\varepsilon>1$ is necessary if $q \neq 2$. We note that in [13] dealing with the nonlinear problem in exterior domains no dependence of a priori estimates on $\frac{1}{|\omega|}$ occurs; the reason is that the author uses strong and weak a priori $L^{2}$-estimates of $u$ by assuming that even $f \in L^{6 / 5}\left(\mathbb{R}^{3}\right) \subset \hat{W}^{-1,2}\left(\mathbb{R}^{3}\right)$. Using results of a forthcoming paper [3] dealing with the weak $L^{q}$-theory of (2) it is obvious that $\|\nu \nabla u\|_{q}$ is bounded uniformly w.r.t. $\omega$ and $k$ by suitable norms of $f$.

TheOREM 4. (1) Let $1<q<2, \nu>0, k>0, \omega=(0,0, \tilde{\omega})^{T} \in \mathbb{R}^{3} \backslash\{0\}$, and let $f \in L^{q}\left(\mathbb{R}^{3}\right)^{3}$. Then (2) has a solution $u \in \hat{W}^{2, q}\left(\mathbb{R}^{3}\right)^{3}$ satisfying the a priori estimates

$$
\left\|\frac{\nabla u}{\left|x^{\prime}\right|}\right\|_{q} \leq \frac{c}{\nu}\|f\|_{q}
$$


and

$$
\left\|\frac{(\omega \wedge x) \cdot \nabla u-\omega \wedge u}{1+\left|x^{\prime}\right|}\right\|_{q} \leq c\left(1+\frac{k}{\nu}\right)\|f\|_{q}
$$

with a constant $c>0$ independent of $k, \nu, \omega ;$ here, for $x=\left(x_{1}, x_{2}, x_{3}\right) \in \mathbb{R}^{3}$ the term $\left|x^{\prime}\right|$ denotes the Euclidean length $\sqrt{x_{1}^{2}+x_{2}^{2}}$ of $x^{\prime}=\left(x_{1}, x_{2}\right)$.

(2) If $1<q<3$, then

$$
\left\|\frac{\nabla u}{|x|}\right\|_{q} \leq \frac{1}{\nu}\|f\|_{q}
$$

and

$$
\left\|\frac{(\omega \wedge x) \cdot \nabla u-\omega \wedge u}{1+|x|}\right\|_{q} \leq c\left(1+\frac{k}{\nu}\right)\|f\|_{q}
$$

with a constant $c>0$ independent of $k, \nu, \omega$.

(3) For all $1<q<\infty$ the third component $u_{3}$ of the solution $u$ satisfies the a priori estimate

$$
\left\|\frac{k \partial_{3} u_{3}}{1+\left|x^{\prime}\right|}\right\|_{q}+\left\|\frac{(\omega \wedge x) \cdot \nabla u_{3}}{1+\left|x^{\prime}\right|}\right\|_{q} \leq c\left(1+\frac{k}{\nu}\right)\|f\|_{q} .
$$

At the end of Section 2 we present a heuristic argument why $L^{q}$-estimates of $k \partial_{3} u$ and of $(\omega \wedge x) \cdot \nabla u-\omega \wedge u$ independent of $\frac{k^{2}}{\nu|\omega|}$ are unlikely to hold and why weighted estimates with the weight $\frac{1}{1+\left|x^{\prime}\right|}$ will help.

2. Preliminaries and proofs. From [2] we recall the calculation of the explicit representation of the solution $u$ of (2). First, we eliminate the pressure term by applying $\operatorname{div}$ to $(2)_{1}$. Then $\nabla p$ is seen to be the unique weak solution of the equation $\Delta p=\operatorname{div} f$ satisfying the a priori estimate

$$
\|\nabla p\|_{q} \leq c\|f\|_{q}
$$

Hence $u$ is the solenoidal solution of the equation

$$
-\nu \Delta u+k \partial_{3} u-(\omega \wedge x) \cdot \nabla u+\omega \wedge u=f-\nabla p
$$

where $f-\nabla p$ is solenoidal. For simplicity, we will write $f$ instead of $f-\nabla p$ and divide by $\tilde{\omega}=|\omega|>0$ to get

$$
-\frac{\nu}{|\omega|} \Delta u+\frac{k}{|\omega|} \partial_{3} u-\left(e_{3} \wedge x\right) \cdot \nabla u+e_{3} \wedge u=\frac{1}{|\omega|} f .
$$

Next use cylindrical coordinates $\left(r, \theta, x_{3}\right) \in \overline{\mathbb{R}_{+}} \times[0,2 \pi) \times \mathbb{R}, r=\left|x^{\prime}\right|=\sqrt{x_{1}^{2}+x_{2}^{2}}$, for $x=\left(x_{1}, x_{2}, x_{3}\right)^{T}$ and observe that

$$
\partial_{\theta} u=\left(e_{3} \wedge x\right) \cdot \nabla u=\left(-x_{2}, x_{1}\right) \cdot \nabla^{\prime} u .
$$

Moreover, we introduce the Fourier transform

$$
\mathcal{F} u(\xi)=\hat{u}(\xi)=(2 \pi)^{-3 / 2} \int_{\mathbb{R}^{3}} e^{-i x \cdot \xi} u(x) d x, \quad \xi \in \mathbb{R}^{3} .
$$


For the Fourier variable $\xi=\left(\xi_{1}, \xi_{2}, \xi_{3}\right) \in \mathbb{R}^{3}$ we also use cylindrical coordinates $\left(s, \varphi, \xi_{3}\right) \in$ $\overline{\mathbb{R}_{+}} \times[0,2 \pi) \times \mathbb{R}, s=\left|\xi^{\prime}\right|=\sqrt{\xi_{1}^{2}+\xi_{2}^{2}}$, and note that

$$
\widehat{\partial_{\theta} u}=\partial_{\varphi} \hat{u}
$$

Thus $\hat{u}$ satisfies the equation

$$
\frac{1}{|\omega|}\left(\nu|\xi|^{2}+i k \xi_{3}\right) \hat{u}-\partial_{\varphi} \hat{u}+e_{3} \wedge \hat{u}=\frac{1}{|\omega|} \hat{f} .
$$

This inhomogeneous, linear ordinary differential equation of first order with respect to $\varphi$ has a unique $2 \pi$-periodic solution. An elementary calculation leads to the representation

$$
\hat{u}(\xi)=\frac{1 /|\omega|}{D(\xi)} \int_{0}^{2 \pi} e^{-\left(\nu|\xi|^{2}+i k \xi_{3}\right) t /|\omega|} O^{T}(t) \hat{f}(O(t) \xi) d t
$$

where

$$
D(\xi)=1-e^{-2 \pi\left(\nu|\xi|^{2}+i k \xi_{3}\right) /|\omega|} .
$$

Moreover, using the geometric series and the $\frac{2 \pi}{|\omega|}$-periodicity of $t \mapsto O_{\omega}^{T}(t) \hat{f}\left(O_{\omega}(t) \xi\right)$ w.r.t. $t$, we get the second representation

$$
\hat{u}(\xi)=\int_{0}^{\infty} e^{-\left(\nu|\xi|^{2}+i k \xi_{3}\right) t} O_{\omega}^{T}(t) \hat{f}\left(O_{\omega}(t) \xi\right) d t .
$$

Note that in $x$-space (18) leads to the identity

$$
u(x)=\int_{0}^{\infty} E_{t} * O_{\omega}^{T}(t) f\left(O_{\omega}(t) \cdot-k t e_{3}\right)(x) d t
$$

where $E$ denotes the heat kernel $E_{t}(x)=\frac{1}{(4 \pi \nu t)^{3 / 2}} e^{-|x|^{2} / 4 \nu t}$ in $\mathbb{R}^{3}$.

Proof of Theorem 2. We start with the case $q=2$ in which we may use the Theorem of Plancherel to estimate $\left\|k \partial_{3} u\right\|_{2}$. By (16), (17), the inequality of Cauchy-Schwarz and Fubini's Theorem,

$$
\begin{aligned}
\int\left|i k \xi_{3} \hat{u}\right|^{2} d \xi & =\int \frac{k^{2} \xi_{3}^{2} /|\omega|^{2}}{|D(\xi)|^{2}}\left|\int_{0}^{2 \pi} e^{-\left(\nu|\xi|^{2}+i k \xi_{3}\right) t /|\omega|} O^{T}(t) \hat{f}(O(t) \xi) d t\right|^{2} d \xi \\
& \leq \int \frac{k^{2} \xi_{3}^{2} /|\omega|^{2}}{|D(\xi)|^{2}}\left(\int_{0}^{2 \pi} e^{-\nu|\xi|^{2} t /|\omega|}|\hat{f}(O(t) \xi)|^{2} d t\right)\left(\int_{0}^{2 \pi} e^{-\nu|\xi|^{2} t /|\omega|} d t\right) d \xi \\
& =\int_{0}^{2 \pi}\left(\int e^{-\nu|\xi|^{2} t /|\omega|} \frac{k^{2} \xi_{3}^{2} /|\omega|^{2}}{|D(\xi)|^{2}} \frac{1-e^{-2 \pi \nu|\xi|^{2} /|\omega|}}{\nu|\xi|^{2} /|\omega|}|\hat{f}(O(t) \xi)|^{2} d \xi\right) d t .
\end{aligned}
$$

In the inner integral the change of variable formula implies that the term $|\hat{f}(O(t) \xi)|^{2}$ may be replaced by $|\hat{f}(\xi)|^{2}$. Then a further application of Fubini's theorem yields

$$
\begin{aligned}
\int\left|i k \xi_{3} \hat{u}\right|^{2} d \xi & =\int|\hat{f}(\xi)|^{2} \frac{k^{2} \xi_{3}^{2} /|\omega|^{2}}{|D(\xi)|^{2}} \frac{1-e^{-2 \pi \nu|\xi|^{2} /|\omega|}}{\nu|\xi|^{2} /|\omega|} \int_{0}^{2 \pi} e^{-\nu|\xi|^{2} t /|\omega|} d t \\
& =\int|\hat{f}(\xi)|^{2} \frac{k^{2} \xi_{3}^{2} /|\omega|^{2}}{|D(\xi)|^{2}} \frac{\left(1-e^{-2 \pi \nu|\xi|^{2} /|\omega|}\right)^{2}}{\nu^{2}|\xi|^{4} /|\omega|^{2}} d \xi .
\end{aligned}
$$


Hence it suffices to consider the 'multiplier function'

$$
m(\xi)=\frac{1-e^{-\left(2 \pi \nu|\xi|^{2} /|\omega|\right.}}{\nu|\xi|^{2} /|\omega|} \frac{k \xi_{3} /|\omega|}{D(\xi)}
$$

and to prove the estimate

$$
|m(\xi)| \leq c\left(1+\frac{k^{2}}{\nu|\omega|}\right), \quad \xi \in \mathbb{R}^{3} .
$$

If $\frac{\nu|\xi|^{2}}{|\omega|}>1$, then $|D(\xi)|$ is bounded below by a positive constant and

$$
|m(\xi)| \leq c \frac{k \xi_{3} /|\omega|}{\nu|\xi|^{2} /|\omega|} \leq c \frac{k}{\nu|\xi|} \leq c \frac{k}{\sqrt{\nu|\omega|}}
$$

If $\frac{\nu|\xi|^{2}}{|\omega|} \leq 1$, the first factor in the definition of $m(\xi)$ is uniformly bounded. To estimate the remaining term

$$
m_{0}(\xi)=\frac{k \xi_{3} /|\omega|}{D(\xi)}
$$

we partition the ball $\frac{\nu|\xi|^{2}}{|\omega|} \leq 1$ into infinitely many slices $S_{n}=\left\{\xi \in \mathbb{R}^{3}: \frac{\nu|\xi|^{2}}{|\omega|} \leq\right.$ 1, $\left.\left|\frac{k \xi_{3}}{|\omega|}-n\right| \leq \frac{1}{4}\right\}, n \in \mathbb{Z}$, and the remaining part $S^{\prime}$ where $\operatorname{dist}\left(\frac{k \xi_{3}}{|\omega|}, \mathbb{Z}\right) \geq \frac{1}{4}$ and consequently $|D(\xi)| \geq 1$. Hence,

$$
\left|m_{0}(\xi)\right| \leq \frac{k\left|\xi_{3}\right|}{|\omega|} \leq \frac{k}{\sqrt{\nu|\omega|}} \quad \text { on } \quad S^{\prime}
$$

For $\xi \in S_{n}, n \in \mathbb{Z}$, Taylor's expansion of $1-e^{-z}$ yields the lower bound

$$
|D(\xi)|=\left|1-e^{-2 \pi\left(\nu|\xi|^{2} /|\omega|+i\left(k \xi_{3} /|\omega|-n\right)\right)}\right| \geq c_{0}\left|\frac{\nu|\xi|^{2}}{|\omega|}+i\left(\frac{k \xi_{3}}{|\omega|}-n\right)\right|
$$

with a constant $c_{0}>0$ independent of all variables $\nu, \xi, k, \omega, n$. Hence for $\xi \in S_{0}$ we get the estimate $\left|m_{0}(\xi)\right| \leq \frac{1}{c_{0}}$. If $\xi \in S_{n}, n \neq 0$, then

$$
\left|m_{0}(\xi)\right| \leq \frac{k\left|\xi_{3}\right| /|\omega|}{\nu|\xi|^{2} /|\omega|} \leq \frac{k}{\nu|\xi|} \leq \frac{4}{3} \frac{k^{2}}{\nu|\omega|}
$$

since $\frac{k|\xi|}{|\omega|} \geq \frac{k\left|\xi_{3}\right|}{|\omega|} \geq \frac{3}{4}$. Now (21) is proved and implies the estimate

$$
\int\left|i k \xi_{3} \hat{u}\right|^{2} d \xi \leq c\left(1+\frac{k^{2}}{\nu|\omega|}\right)^{2} \int|\hat{f}|^{2} d \xi
$$

Then the Theorem of Plancherel completes the proof in the case $q=2$.

For the case $q \neq 2$ we write $(16)$ in the form

$$
\hat{u}(\xi)=\frac{1}{|\omega|} \int_{0}^{2 \pi} \frac{1}{D(\xi)} e^{-\nu|\xi|^{2} t /|\omega|} O^{T}(t)\left(\mathcal{F} f\left(O(t) \cdot-k t e_{3} /|\omega|\right)\right)(\xi) d t
$$

using that $e^{-i t k \xi_{3}} \in \mathcal{S}^{\prime}\left(\mathbb{R}^{3}\right)$ is the Fourier transform of the shift operator $f \mapsto f\left(\cdot-k t e_{3}\right)$ on $\mathcal{S}^{\prime}\left(\mathbb{R}^{3}\right)$. Hence in $x$-space,

$$
k \partial_{3} u(x)=\int_{0}^{2 \pi} T_{t} F(t, \cdot)(x) d t
$$


where

$$
F(t, \cdot)=O^{T}(t) f\left(O(t) \cdot-k t e_{3} /|\omega|\right)
$$

and the operator family $T_{t}, 0<t<2 \pi$, is defined by its multiplier

$$
m_{t}(\xi)=\frac{i k \xi_{3} /|\omega|}{D(\xi)} e^{-\nu|\xi|^{2} t /|\omega|},
$$

i.e., $T_{t}=\mathcal{F}^{-1}\left(m_{t}(\xi) \cdot\right)$. Note that $\|F(t, \cdot)\|_{q} \leq\|f\|_{q}$ for all $t \in(0,2 \pi)$.

Below we will prove the multiplier estimate

$$
\max _{\alpha} \sup _{\xi \neq 0}\left|\xi^{\alpha} D_{\xi}^{\alpha} m_{t}(\xi)\right| \leq c\left(1+\frac{k}{\sqrt{\nu|\omega| t}}+\frac{k^{2}}{\nu|\omega|}\right) \cdot\left(1+\frac{k^{2}}{\nu|\omega|}\right)
$$

with a constant $c>0$ independent of $\nu, \omega, k$ and $t$; here $\alpha \in \mathbb{N}_{0}^{3}$ runs through the set of all multi-indices $\alpha \in\{0,1\}^{3}$. Then Marcinkiewicz' multiplier theorem [14] implies that in the operator norm $\|\cdot\|_{q}$ on $L^{q}$

$$
\left\|T_{t}\right\|_{q} \leq c\left(1+\frac{k}{\sqrt{\nu|\omega| t}}+\frac{k^{2}}{\nu|\omega|}\right) \cdot\left(1+\frac{k^{2}}{\nu|\omega|}\right) .
$$

Hence

$$
\left\|k \partial_{3} u\right\|_{q} \leq c \int_{0}^{2 \pi}\left\|T_{t}\right\|_{q}\|F(t, \cdot)\|_{q} d t \leq c\left(1+\frac{k^{4}}{\nu^{2}|\omega|^{2}}\right)\|f\|_{q}
$$

with a constant $c>0$ independent of $\nu, \omega$ and $k$.

Now the $L^{2}$-result and (24) are combined by using complex multiplier theory to prove (6). Given $1<q<2$ we formally interpolate between the $L^{2}$-result and the $L^{1}$-result (24) using $\theta=\frac{2}{q}-1$ such that $\frac{1}{q}=\frac{1-\theta}{2}+\frac{\theta}{1}$. Then

$$
\left\|\partial_{3} u\right\|_{q} \leq c\left(1+\frac{k^{2}}{\nu|\omega|}\right)^{2-2 / q}\left(1+\frac{k^{4}}{\nu^{2}|\omega|^{2}}\right)^{2 / q-1}\|f\|_{q}
$$

yielding $\left\|\partial_{3} u\right\|_{q} \leq c\left(1+\frac{k^{2}}{\nu|\omega|}\right)^{2 / q}\|f\|_{q}$. Since no estimate (24) holds for $L^{1}$, we have to interpolate between $L^{2}$ and $L^{p}$ for $p>1$ arbitrarily close to 1 . Therefore, the additional exponent $\varepsilon>0$ in (6) occurs. If $2<q<\infty$, we formally interpolate between $L^{2}$ and $L^{\infty}$ to get (6) with the exponent $2(1-1 / q)$. Since again there is no $L^{\infty}$-result available, we choose $p$ arbitrarily large instead of $p=\infty$ and have to add the exponent $\varepsilon>0$ in (6).

Finally we prove $(23)$, start with $m_{t}$ itself and distinguish between the cases $\frac{\nu|\xi|^{2}}{|\omega|}>1$ and $\frac{\nu|\xi|^{2}}{|\omega|} \leq 1$. In the first case $|D(\xi)|$ is bounded below by a positive constant yielding

$$
\left|m_{t}(\xi)\right| \leq c \frac{k\left|\xi_{3}\right|}{|\omega|} e^{-\nu|\xi|^{2} t /|\omega|} \leq c \frac{k}{\sqrt{\nu|\omega| t}} .
$$

If $\frac{\nu|\xi|^{2}}{|\omega|} \leq 1$, we may neglect the term $e^{-\nu|\xi|^{2} t /|\omega|}$ and conclude from the detailed estimates of $m_{0}(\xi)$ in the $L^{2}$-case above that

$$
\left|m_{t}(\xi)\right| \leq\left|m_{0}(\xi)\right| \leq c\left(1+\frac{k^{2}}{\nu|\omega|}\right) .
$$


Hence $\left|m_{t}(\xi)\right| \leq c\left(1+\frac{k}{\sqrt{\nu|\omega| t}}+\frac{k^{2}}{\nu|\omega|}\right)$ proving (23) for $m_{t}$. Next consider

$$
\xi_{3} \partial_{3} m_{t}(\xi)=m_{t}(\xi)-\frac{2 \nu \xi_{3}^{2} t}{|\omega|} m_{t}(\xi)-\xi_{3} \frac{\partial_{3} D(\xi)}{D(\xi)} m_{t}(\xi)
$$

where

$$
\xi_{3} \frac{\partial_{3} D(\xi)}{D(\xi)}=2 \pi \frac{\left(2 \nu \xi_{3}^{2}+i k \xi_{3}\right) /|\omega|}{D(\xi)} e^{-2 \pi\left(\nu|\xi|^{2}+i k \xi_{3}\right) /|\omega|} .
$$

Writing the exponential function $e^{-\nu|\xi|^{2} t /|\omega|}$ as $e^{-\nu|\xi|^{2} t / 2|\omega|} \cdot e^{-\nu|\xi|^{2} t / 2|\omega|}$, we see that the second term on the right-hand side of (25) may be estimated as $m_{t}$ itself. In the third term note - due to properties of $D(\xi)$ proved above - that $\frac{2 \nu \xi_{3}^{2} /|\omega|}{D(\xi)} e^{-2 \pi\left(\nu|\xi|^{2}+i k \xi_{3}\right) /|\omega|}$ is uniformly bounded. Moreover, the estimate of $\frac{k \xi_{3} /|\omega|}{D(\xi)} e^{-2 \pi\left(\nu|\xi|^{2}+i k \xi_{3}\right) /|\omega|}$ is similar to the estimate of the multiplier function $m(\xi)$ in (20), (21) yielding

$$
\frac{k\left|\xi_{3}\right| /|\omega|}{D(\xi)} e^{2 \pi\left(-\nu|\xi|^{2}+i k \xi_{3}\right) /|\omega|} \leq c\left(1+\frac{k^{2}}{\nu|\omega|}\right) .
$$

Combining the previous estimates we get (23) for $\xi_{3} \partial_{3} m_{t}$. Concerning $\xi_{1} \partial_{1} m_{t}(\xi)$ note that (26) must be replaced by

$$
\xi_{1} \frac{\partial_{1} D(\xi)}{D(\xi)}=2 \pi \frac{2 \nu \xi_{1}^{2} /|\omega|}{D(\xi)} e^{-2 \pi\left(\nu|\xi|^{2}+i k \xi_{3}\right) /|\omega|} .
$$

Obviously, looking at the properties of $D(\xi)$ proved above, the modulus of this term is uniformly bounded requiring no further power of $k^{2} / \nu|\omega|$. Since the same assertion holds for the derivatives $\xi_{2} \partial_{2}$ and $\xi_{1} \partial_{1} \xi_{2} \partial_{2}$ of $m_{t},(23)$ is completely proved.

EXAmple 3. For fixed $k>0, \nu>0$ we will construct a sequence of solenoidal forces $(f)=\left(f_{\omega}\right) \subset L^{2}\left(\mathbb{R}^{3}\right)^{3},|\omega| \rightarrow 0$, such that the corresponding sequence of solutions $(u)=$ $\left(u_{\omega}\right)$ will satisfy

$$
\left\|k \partial_{3} u\right\|_{q} \geq c \frac{k^{2}}{\nu|\omega|}\|f\|_{q}
$$

with $c>0$ independent of $k, \nu, \omega$. Given $k>0, \nu>0$ choose $|\omega|$ small enough such that

$$
\frac{\nu|\omega|}{k^{2}}<\frac{1}{16}
$$

Then define $f=\left(f^{\prime}, 0\right) \in L^{2}\left(\mathbb{R}^{3}\right)^{3}$ such that in Fourier space

$$
\hat{f}^{\prime}(\xi)=i\left\{\begin{array}{l}
\xi^{\prime \perp}, 0<\varphi<\pi \\
-\xi^{\prime \perp}, \pi<\varphi<2 \pi
\end{array}, \quad \text { when } \quad\left|\frac{k\left|\xi^{\prime}\right|}{|\omega|}-1\right|<\frac{1}{2},\left|\frac{k\left|\xi_{3}\right|}{|\omega|}-1\right|<\frac{1}{2}\right.
$$

but $\hat{f}^{\prime}(\xi)=0$ elsewhere; here, as usual, $\varphi$ is the angular part of $\xi$. Since $\overline{\hat{f}(\xi)}=\hat{f}(-\xi)$, the vector field $f$ is real-valued and obviously solenoidal. By (16)

$$
\hat{u}(\xi)=\frac{e^{\left.\nu|\xi|^{2}+i k \xi_{3}\right) \varphi /|\omega|}}{D(\xi)|\omega|} O(\varphi) \int_{\varphi}^{\varphi+2 \pi} e^{-\left(\nu|\xi|^{2}+i k \xi_{3}\right) t /|\omega|} O^{T}(t) \hat{f}\left(O(t) e_{1}\right) d t .
$$


Since $O^{T}(t) \hat{f}\left(O(t) e_{1}\right)=+e_{2}$ and $=-e_{2}$ when $0<\varphi<\pi$ and $\pi<\varphi<2 \pi$, resp., a simple integration leads to the formula

$$
i k \xi_{3} \hat{u}(\xi)=\hat{f}(\xi) \frac{i k \xi_{3} /|\omega|}{\left(\nu|\xi|^{2}+i k \xi_{3}\right) /|\omega|}\left(1-\frac{2 e^{-(\pi-\varphi)\left(\nu|\xi|^{2}+i k \xi_{3}\right) /|\omega|}}{1+e^{-\pi\left(\nu|\xi|^{2}+i k \xi_{3}\right) /|\omega|}}\right),
$$

when $0<\varphi<\pi$; for $\pi<\varphi<2 \pi$ the exponential term $e^{-(\pi-\varphi)\left(\nu|\xi|^{2}+i k \xi_{3}\right) /|\omega|}$ must be replaced by $e^{-(2 \pi-\varphi)\left(\nu|\xi|^{2}+i k \xi_{3}\right) /|\omega|}$. The assumptions on $k, \nu, \omega$ imply for $\xi \in \operatorname{supp} \hat{f}$ that $\left|\frac{k \xi_{3}}{|\omega|}\right| \sim 1, \quad \frac{\nu|\xi|^{2}}{|\omega|} \sim \frac{\nu|\omega|}{k^{2}}$; consequently, we have $\left.|\nu| \xi\right|^{2}+i k \xi_{3}|/| \omega|\sim| \frac{k \xi_{3}}{|\omega|} \mid \sim 1$ and $\left|e^{-(\pi-\varphi)\left(\nu|\xi|^{2}+i k \xi_{3}\right) /|\omega|}\right| \sim 1$. Finally, the crucial term is

$$
\left|1+e^{-\pi\left(\nu|\xi|^{2}+i k \xi_{3}\right) /|\omega|}\right| \sim \frac{\nu|\xi|^{2}}{|\omega|} \sim \frac{\nu|\omega|}{k^{2}} \text { for } \xi \in \operatorname{supp} \hat{f} .
$$

Hence

$$
\left|i k \xi_{3} \hat{u}(\xi)\right| \sim \frac{k^{2}}{\nu|\omega|}|\hat{f}(\xi)| \quad \text { for } \xi \in \operatorname{supp} \hat{f} .
$$

Since all similarity estimates $\sim$ can be made precise by using positive constants independent of $k, \nu, \omega,(27)$ is proved.

Proof of Theorem 4. (i) Given a solution $u \in \hat{W}^{2, q}\left(\mathbb{R}^{3}\right)^{3}$, i.e. a function $u$ with $\nabla^{2} u \in$ $L^{q}\left(R^{3}\right)$, Theorems 1 and 2 yield $\beta \in \mathbb{R}$ such that $\nabla^{\prime}(u-\beta(\omega \wedge x)) \in L^{r}\left(\mathbb{R}^{3}\right)^{6}, \frac{1}{r}=\frac{1}{q}-\frac{1}{4}$, and $\partial_{3}(u-\beta(\omega \wedge x)) \in L^{q}\left(\mathbb{R}^{3}\right)^{3}$. Since also $u-\beta \omega \wedge x$ solves (2), assume without loss of generality that $\beta=0$ implying for a.a. $x_{3} \in \mathbb{R}$ that

$$
\int_{\mathbb{R}^{2}}\left|\nabla^{\prime} u\left(x^{\prime}, x_{3}\right)\right|^{r} d x^{\prime}<\infty, \quad \int_{\mathbb{R}^{2}}\left|\partial_{3} u\left(x^{\prime}, x_{3}\right)\right|^{q} d x^{\prime}<\infty .
$$

Then classical arguments show the existence of a sequence of radii $\left(R_{j}\right) \subset \mathbb{R}_{+}$such that

$$
\int_{0}^{2 \pi}\left|\nabla^{\prime} u\left(R_{j}, \theta, x_{3}\right)\right|^{r} d \theta=o\left(R_{j}^{-2}\right), \quad \int_{0}^{2 \pi}\left|\partial_{3} u\left(R_{j}, \theta, x_{3}\right)\right|^{q} d \theta=o\left(R_{j}^{-2}\right)
$$

as $j \rightarrow \infty$.

On the other hand, since $1<q<2$ and $\nabla^{2} u \in L^{q}\left(\mathbb{R}^{3}\right)$, Theorem II5.1 in [6] yields for a.a. $x_{3} \in \mathbb{R}$ a matrix $A\left(x_{3}\right) \in \mathbb{R}^{3,3}$ such that

$$
\left(\int_{\mathbb{R}^{2}} \frac{\left|\nabla u\left(x^{\prime}, x_{3}\right)-A\left(x_{3}\right)\right|^{q}}{\left|x^{\prime}\right|^{q}} d x^{\prime}\right)^{1 / q} \leq \frac{q}{2-q}\left(\int_{\mathbb{R}^{2}}\left|\nabla^{\prime} \nabla u\left(x^{\prime}, x_{3}\right)\right|^{q} d x^{\prime}\right)^{1 / q} .
$$

Note that Theorem II5.1 in [6] is stated only for exterior domains; however, since the constant $q /(2-q)$ does not depend on the 'inner radius' of the exterior domain, the estimate holds for the whole space $\mathbb{R}^{2}$ as well. Moreover, by Lemma 5.2 in [6],

$$
\int_{0}^{2 \pi}\left|\nabla u\left(R, \theta, x_{3}\right)-A\left(x_{3}\right)\right|^{q} d \theta=o\left(R^{q-2}\right)
$$

as $R \rightarrow \infty$. Now (28), (30) show that $A\left(x_{3}\right)=0$; hence (29) and (3) yield (7). Then (8) is an easy consequence of (7) and of $(2)$.

(ii) If $1<q<3$, Theorem II5.1 in [6] yields the estimate

$$
\left(\int_{\mathbb{R}^{3}} \frac{|\nabla u(x)-A|^{q}}{|x|^{q}} d x\right)^{1 / q} \leq \frac{q}{3-q}\left(\int_{\mathbb{R}^{3}}\left|\nabla^{2} u(x)\right|^{q} d x\right)^{1 / q}
$$


with a constant matrix $A \in \mathbb{R}^{3,3}$. Moreover, by Lemma 5.2 in [6],

$$
\int_{|y|=1}|\nabla u(R y)|^{q} d o(y)=o\left(R^{q-3}\right)
$$

as $R \rightarrow \infty$, where $\int_{|y|=1} \ldots d o(y)$ denotes the surface integral on the unit sphere of $\mathbb{R}^{3}$. Since $\nabla^{\prime} u \in L^{r}\left(\mathbb{R}^{3}\right)^{6}$ and $\partial_{3} u \in L^{q}\left(\mathbb{R}^{3}\right)^{3}$, arguments as above imply that $A$ vanishes. Now (9) and (10) are easy consequences.

(iii) By (2) $u_{3}$ solves the problem $-\nu \Delta u_{3}+k \partial_{3} u_{3}-(\omega \wedge x) \cdot \nabla u_{3}=f_{3}$. Since $(\omega \wedge x) \cdot \nabla u_{3}=|\omega| \partial_{\theta} u_{3}$, an integration w.r.t. $\theta \in(0,2 \pi)$ yields for the $\theta$-independent function $U_{3}(x):=\frac{1}{2 \pi} \int_{0}^{2 \pi} u_{3}\left(\left|x^{\prime}\right|, \theta^{\prime}, x_{3}\right) d \theta^{\prime}$ the equation

$$
-\nu \Delta U_{3}+k \partial_{3} U_{3}=\frac{1}{2 \pi} \int_{0}^{2 \pi} f_{3} d \theta^{\prime} .
$$

Applying Fourier transforms and using Marcinkiewicz' multiplier theorem we get that $U_{3}$ satisfies the estimate

$$
\left\|k \partial_{3} U_{3}\right\|_{q} \leq c\|f\|_{q}
$$

with a constant $c>0$ independent of $f, k, \nu$, cf. the analysis of the related Oseen problem [1], [5], [6]. By Wirtinger's inequality there exists a constant $c>0$ such that for a.a. $r=\left|x^{\prime}\right|>0$ and $x_{3} \in \mathbb{R}$

$$
\left\|\partial_{3} u_{3}\left(r, \cdot, x_{3}\right)\right\|_{L^{q}(0,2 \pi)} \leq c\left(\left\|\partial_{\theta} \partial_{3} u_{3}\left(r, \cdot, x_{3}\right)\right\|_{L^{q}(0,2 \pi)}+\left\|\partial_{3} U_{3}\left(r, \cdot, x_{3}\right)\right\|_{L^{q}(0,2 \pi)}\right) .
$$

Now divide by $1+r$ and integrate w.r.t. $r d r, r>0$, and $d x_{3}, x_{3} \in \mathbb{R}$, to get that

$$
\left\|\frac{\partial_{3} u_{3}}{1+\left|x^{\prime}\right|}\right\|_{q} \leq c\left(\left\|\frac{\partial_{\theta} \partial_{3} u_{3}}{1+\left|x^{\prime}\right|}\right\|_{q}+\left\|\frac{\partial_{3} U_{3}}{1+\left|x^{\prime}\right|}\right\|_{q}\right) \text {. }
$$

Since the second term on the right-hand side is bounded by $\left\|\partial_{3} \nabla^{\prime} u_{3}\right\|_{q} \leq(c / \nu)\|f\|_{q}$ and since the third term is bounded by $\left\|\partial_{3} U_{3}\right\|_{q} \leq(c / k)\|f\|_{q}$, we get (11).

REMARK. The ideas of the proof of Theorem 4 (iii) do not apply to $u_{1}$ and $u_{2}$, since the term $\omega \wedge u$ does not vanish when applying the integration $\int_{0}^{2 \pi} \ldots d \theta^{\prime}$. Also the identity $(\omega \wedge x) \cdot \nabla u-\omega \wedge u=|\omega| O(\theta) \partial_{\theta}\left(O^{T}(\theta) u\right)$ will not help, since no a priori estimates of $\partial_{3} \partial_{\theta}\left(O^{T}(\theta) u\right)$ are available except for the case when $1<q<2$.

Heuristic argument. Let us motivate why estimates of $(\omega \wedge x) \cdot \nabla u-\omega \wedge u$ and of $k \partial_{3} u$ cannot be expected to be independent of $k^{2} / \nu|\omega|$. For simplicity ignore the terms $\omega \wedge u$ and $p$, recall that $(\omega \wedge x) \cdot \nabla u=|\omega| \partial_{\theta} u$ and let us perform a simple scaling analysis. Define the non-dimensional quantities $\tilde{u}=|\omega| u / A$, where $A \in \mathbb{R}$ is a characteristic acceleration

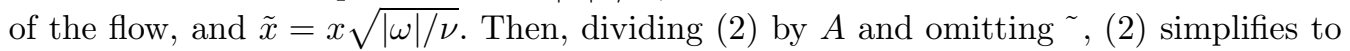
the non-dimensional equation

$$
-\Delta u+\frac{k}{\sqrt{\nu|\omega|}} \partial_{3} u-\partial_{\theta} u=f \quad \text { in } \mathbb{R}^{3} .
$$

Note that $\frac{k}{\sqrt{\nu|\omega|}}$ is a new non-dimensional characteristic number of the flow. For fixed $r=\left|x^{\prime}\right|$ let us interpret $\frac{k}{\sqrt{\nu|\omega|}} \partial_{3} u-\partial_{\theta} u$ as a directional derivative defined by the unit 
vector

$$
d_{\omega}\left(x^{\prime}\right)=\frac{1}{\sqrt{r^{2}+k^{2} / \nu \omega}}\left(\frac{k}{\sqrt{\nu|\omega|}} e_{3}-\left(-x_{2}, x_{1}, 0\right)^{T}\right) \in \mathbb{R}^{3}
$$

which is tangential to the cylinder $\mathcal{C}_{r}=\left\{x \in \mathbb{R}^{3}:\left|x^{\prime}\right|=r\right\}$. Hence, defining the curve

$$
\gamma_{\omega}(s)=\left(-r \cos s,-r \sin s, \frac{k}{\sqrt{\nu|\omega|}} s\right)^{T}
$$

on $\mathcal{C}_{r}$ with tangential vector $\sqrt{r^{2}+k^{2} / \nu \omega} d_{\omega}$, we get that

$$
\frac{d}{d s} u\left(\gamma_{\omega}(s)\right)=\left(\sqrt{r^{2}+k^{2} / \nu \omega} d_{\omega} \cdot \nabla u\right)\left(\gamma_{\omega}(s)\right) .
$$

Obviously $d_{\omega}$ converges to the third unit vector $e_{3}$, whereas the curve $\gamma_{\omega}(s)$ has no reasonable limit on the cylinder $\mathcal{C}_{r}$. In this sense, the information on the directional derivative $d_{\omega} \cdot \nabla u$ on $\mathcal{C}_{r}$ is lost in the limit $\omega=0$. This discrepancy vanishes for $r=0$, but gets larger as $r \rightarrow \infty$. Therefore, the weight $\frac{1}{1+\left|x^{\prime}\right|}$ has to occur in Theorem 1.4.

\section{References}

[1] R. Farwig, The stationary Navier-Stokes equations in a 3D-exterior domain. in: Recent Topics on Mathematical Theory of Viscous Incompressible Fluid, Lecture Notes in Num. Appl. Anal. 16 (1998), 53-115.

[2] R. Farwig, An $L^{q}$-analysis of viscous fluid flow past a rotating obstacle, Darmstadt University of Technology, Department of Mathematics, Preprint no. 2325 (2004), to appear in Tohoku Math. J.

[3] R. Farwig, Weak solutions of Navier-Stokes flow past a rotating obstacle, in preparation.

[4] R. Farwig, T. Hishida and D. Müller, $L^{q}$-theory of a singular "winding" integral operator arising from fluid dynamics, Pacific J. Math. 215 (2004), 297-312.

[5] R. Farwig and H. Sohr, Weighted estimates for the Oseen equations and the Navier-Stokes equations in exterior domains, in: Theory of Navier-Stokes equations, J. Heywood et al. (eds.), Series Advances Math. Appl. Sciences 47, World Scientific, 1998, 11-31.

[6] G. P. Galdi, An Introduction to the Mathematical Theory of the Navier-Stokes Equations, Vol. I. Linearized Steady Problems, Springer Tracts in Natural Philosophy 38, 2nd edition, 1998.

[7] G. P. Galdi, On the motion of a rigid body in a viscous liquid: A mathematical analysis with applications, in: S. Friedlander and D. Serre (eds.), Handbook of Mathematical Fluid Mechanics, Elsevier Science, 2002, 653-791.

[8] G. P. Galdi, Steady flow of a Navier-Stokes fluid around a rotating obstacle, J. Elasticity 71 (2003), 1-32.

[9] T. Hishida, An existence theorem for the Navier-Stokes flow in the exterior of a rotating obstacle, Arch. Rational Mech. Anal. 150 (1999), 307-348.

[10] T. Hishida, The Stokes operator with rotation effect in exterior domains, Analysis 19 (1999), 51-67.

[11] Š. Nečasova, Some remarks on the steady fall of a body in Stokes and Oseen flow, Acad. Sciences Czech Republic, Math. Institute, Preprint 143 (2001).

[12] Š. Nečasova, Asymptotic properties of the steady fall of a body in viscous fluids, Acad. Sciences Czech Republic, Math. Institute, Preprint 149 (2002). 
[13] A. L. Silvestre, On the existence of steady flows of a Navier-Stokes liquid around a moving body, Math. Meth. Appl. Sci. 27 (2004), 1399-1409.

[14] E. M. Stein, Singular Integrals and Differentiability Properties of Functions, Princeton University Press, Princeton, N.J., 1970. 